\title{
Patterned substrates modulate growth and dynamics of 3D cellular systems
}

\author{
Michael J. Fanous ${ }^{1,2}$, Yanfen Li $^{1,3}$, Mikhail E. Kandel ${ }^{2}$, Kristopher A. Kilian ${ }^{1,4}$, and Gabriel \\ Popescu $^{1,2 *}$
}

1. Department of Bioengineering, University of Illinois at Urbana-Champaign, Urbana, Illinois 61801, USA

2. Quantitative Light Imaging Laboratory, Department of Electrical and Computer Engineering, Beckman Institute for Advanced Science and Technology, University of Illinois at UrbanaChampaign, Urbana, Illinois 61801, USA

3. Department of Biomedical Engineering, University of Massachusetts Lowell, Lowell, Massachusetts 01854, USA

4. School of Chemistry, School of Materials Science and Engineering, Australian Centre for NanoMedicine, University of New South Wales, Sydney, NSW 2052, Australia

\section{*) gpopescu@illinois.edu}

Key words: substrate engineering, organoids, radial transport, dispersion analysis, 3D cellular dynamics, quantitative phase imaging, label free imaging

\begin{abstract}
The development of 3D cellular architectures during development and pathological processes involves intricate migratory patterns that are modulated by genetics and the surrounding microenvironment. The substrate composition of cell cultures has been demonstrated to influence growth, proliferation, and migration in 2D. Here we study the growth and dynamics of mouse embryonic fibroblast (MEF) cultures patterned in a tissue sheet which then exhibits 3D growth. Using gradient light interference microscopy (GLIM), a label-free quantitative phase imaging approach, we explored the influence of geometry on cell growth patterns and rotational dynamics. We apply, for the first time to our knowledge, dispersion-relation phase spectroscopy (DPS) in
\end{abstract}


polar coordinates to generate the radial and rotational cell mass-transport. Our data show that cells cultured on engineered substrates undergo rotational transport in a radially independent manner and exhibit faster vertical growth than the control, unpatterned cells. The use of GLIM and polar DPS provides a novel quantitative approach to studying the effects of spatially patterned substrates on cell motility and growth.

\section{Introduction}

The mechanical properties of a cell's microenvironment play a role in guiding cellular functions, including cell migration and growth patterns[1-3]. Cells sense the extracellular matrix (ECM) via cell surface receptors which then propagate the mechanochemical signals into the cell to influence cellular functions downstream[4]. Cellular behavior can thus be modulated by mechanical[5], biochemical[6, 7], or thermal[8] stimuli. Substrate engineering provides a reproducible approach to study these mechanochemical cues including the ability to control the boundary mechanics and geometry of cells and tissues[9-11]. Micropatterning of large populations of cells produces a gradient of force spatially organized within the pattern which then leads to differing patterns of cellular function[12], including cellular differentiation[13, 14] and migration[15]. Patterning the geometry of culture substrates causes mutations to a cell's intrinsic makeup, which is phenomenon that remains insufficiently understood[10, 15-18]. The 3D dynamics of biological specimens, such as organoids, can be diverse, complex, and challenging to assess precisely[19, 20]. Though several studies have reported on the effects of cell cultures[21], no technique, to our knowledge, has been used to simultaneously tackle quantitative growth and mass transport. 
Typical methods to characterize complex biological specimens involve exogenous contrast agents, such as fluorescence dyes[22, 23]. These artificial inserts affect the cell's original composition and can lead to damage via phototoxicity[24]. A further limitation with fluorescent modalities is the photobleaching effect that restricts imaging duration[25].

Quantitative phase imaging (QPI)[26] is a label-free approach that has emerged as a powerful alternative to measuring biological phenomena quantitatively[27]. QPI's key feature is that it can image cells nondestructively[28, 29]. The recurrent drawback with laser instruments, however, has been the artifact of speckles resulting from coherent light sources[30-32]. During the past decade, several white light based methods have been devised to obtain speckle-free images[33-37] .In particular, Spatial Light Interference Microscopy (SLIM)[26] and Gradient Light Interference Microscopy (GLIM)[37] have been used successfully to study the 3D morphology of various sample types, such single cells[38, 39], embryos[27], red blood cells[40], and neural networks[41]. Very recently, SLIM has been used for high-throughput single cell weight phenotyping in biomass producing cell populations[42]. GLIM is particularly well suited for subduing multiple scattering backgrounds and for effectively reducing out-of-focus light. Both systems employ a spatial light modulator to combine phase shifting and common path interferometry and generate quantitative phase information. SLIM and GLIM are implemented as add-ons to customary phase contrast[43] and differential interference contrast (DIC) microscope stands[44], respectively. Dispersion-relation phase spectroscopy (DPS)[45] has been developed as an analysis method for phase images to inspect diffusion and advection signatures in cellular systems[41, 46-48].

In this paper, we use GLIM in conjunction with a modified version of the DPS technique, to investigate the growth and rotational dynamics of MEF cultures on patterned substrates as they 
grow into 3D aggregates, approximating spheroidal architecture. We observe that substrate patterning induces the cells to exhibit greater vertical growth and that the cells undergo rotational fluctuations in a plane parallel to the substrate. Our methods will likely facilitate a deeper understanding of 3D migration and proliferation processes that underlie cellular assembly into tissue-mimetic structures, during morphogenesis[49].

\section{Methods}

Unless otherwise mentioned, all materials were acquired from Sigma-Aldrich. Tissue culture plastic ware was purchased from VWR. Glass coverslips were purchased from Fisher Scientific. Cell culture media reagents were purchased from Gibco.

\subsection{Cell Culture}

Mouse embryonic fibroblasts (MEFs) were obtained through a generous donation from Dr. Quanxi Li of the Department of Comparative Biosciences, University of Illinois at Urbana-Champaign. The cells were cultured in glucose $(5 \mathrm{~g} / \mathrm{mL})$ DMEM supplemented with $15 \%$ fetal bovine serum (FBS, Invitrogen) and 1\% penicillin/streptomycin. Cells were passaged at $80 \%$ confluency with $0.5 \%$ Trypsin: EDTA and the growth medium was replaced every 3 days. For imaging, cells were seeded at $\sim 200,000$ cells/cm in a 6 well glass bottom plate (P06-20-1.5-N) and were imaged over a duration of 100 hours every 30 minutes with an acquisition rate of 6 frames/s. The microscope housed an incubator unit to sustain the cells at 37 degree Celsius, and 5\% $\mathrm{CO}_{2}$. Wells either had patterned or normal substrate configurations, and each field of view was imaged by a 10-layer zstack of $10 \mu \mathrm{m}$ increments. Surface plots and videos were generated through maximum projection 
renderings. The heights of the sample were determined by computing the peak maximum values of normalized phase gradients in each frame of the z-stack.

\subsection{Gel Preparation}

$10 \mathrm{kPa}$ polyacrylamide hydrogels were produced as previously described to mimic the stiffness of an MEF's natural bioenvironment[50]. In brief terms, a mixture of 5\% polyacrylamide and $0.15 \%$ bis-acylamide was fabricated and then combined with $0.1 \%$ Ammonium Persulfate (APS) and 0.1\% Tetramethylenediamine (TEMED)[51]. Solutions were placed onto a hydrophobically prepared glass slide (Rain-X) and sandwiched between an aminopropyltriethoxisilane (APTES)sinalized glass coverslip. Gels were removed from the coverslip after polymerization and immersed in 55\% hydrazine hydrate (Fisher) for one hour, and then washed with $5 \%$ glacial acetic acid for one hour.

\subsection{Gel Pattering}

Circular SU-8 silicon masters were fashioned using photolithography. To produce stamps, Polydimethysiloxane (PDMS, Polysciences, Inc) was polymerized over the silicon masters[11]. $25 \mu \mathrm{g} / \mathrm{ml}$ fibronectin was incubated with Sodium Periodate for 45 minutes and incubated on top of PDMS stamps for 30 minutes. The stamps were then air dried and applied to the hydrogels to create the desired patterns.

\subsection{Gradient Light Interference Microscopy}

Measurements were performed using GLIM, consisting of an inverted differential interference contrast (DIC) microscope (Axio Observer Z1, Zeiss, in this case) and an add-on module (CellVista GLIM Pro, Phi Optics, Inc). GLIM generates quantitative phase gradient images of the sample with high depth sectioning capability. Quantitative phase methods often use coherent light 
sources that hamper the contrast of the images due to scattering artifacts[37]. GLIM resolved this problem by using low-coherence interferometry with white light, enabling extremely sensitive measurements. GLIM is also label-free, which allows imaging for long durations without imposing deleterious conditions on the cells.

\section{Polar DPS}

To study the translational and radial components of cellular mass transport, we used the dispersionrelation phase spectroscopy (DPS) technique[45] in polar coordinates. This method is effective at distilling spatiotemporal dynamic information from a time-lapse sequence of phase images. It does not require any manual tracing or labelling and enables automatic computations[41]. In DPS, the dispersion relation of the motile system is evaluated, combining the spatial and temporal frequencies. The nature of the dispersion curve informs on the type of transport, whether it is diffusive or deterministic. For our analysis, we first convert the GLIM images into polar coordinates and then perform DPS as previously described[41]. However, instead of taking an azimuthal average of the power spectrum, we evaluated both the rotational and radial dynamics separately. The dry mass-density fluctuations can be described via an advection-diffusion equation as follows,

$$
D \nabla^{2} \rho(\mathbf{r}, t)-\mathbf{v} \cdot \nabla \rho(\mathbf{r}, t)-\partial(\mathbf{r}, t) / \partial t=0
$$

where $\mathbf{r}=(r, \theta)$ indicates the $2 \mathrm{D}$ coordinate, $\mathbf{v}$ is the advection velocity, and $D$ is the diffusion coefficient. Using this equation, we obtain the temporal autocorrelation function computed at every spatial frequency, $\mathbf{r}=(r, \theta)$ and temporal delay, $\tau$, defined as $g(\mathbf{q}, \tau)=\left\langle\tilde{\rho}(\mathbf{q}, t) \stackrel{\tilde{\rho}^{*}}{\rho}(\mathbf{q}, t+\tau)\right\rangle_{t} /\left\langle\left.\tilde{\rho}(\mathbf{q}, t)\right|^{2}\right\rangle_{t}$. Here, $\tilde{\rho}(\mathbf{q}, t)=\mathrm{F}_{\mathbf{q}}[\rho(\mathbf{r}, t)]$ is the 2D spatial Fourier 
transform of the dry mass density. It has been shown previously that one can calculate $g(\mathbf{q}, \tau)$ directly from the phase gradient $\nabla \phi$ instead of the phase itself $\phi$ [37], which yields

$$
g(\mathbf{q}, \tau)=e^{i \mathbf{v}_{0} \cdot \mathbf{q} \tau} e^{-\left(\Delta v \mathbf{q}+D \mathbf{q}^{2}\right) \tau}
$$

where $\mathbf{V}_{0}$ is the mean and $\Delta v$ the standard deviation of the velocity distribution. At each rotational or translational spatial frequency, one can fit the measurement of $g(\mathbf{q}, \tau)$ to compute $\Delta v_{\theta}$ and $\Delta v_{r}$ . The decay rate of $g(\mathbf{q}, \tau)$ is governed by

$$
\Gamma(\mathbf{q})=\Delta v q+D q^{2}
$$

where $\Delta v$ corresponds to the active transport and $D$ refers to diffusion.

\section{Results}

\subsection{Effects of circular substrate patterning on 3D cell growth}

To explore how geometric constraints influence the spatiotemporal development of large populations of cells, mouse embryonic fibroblasts (MEFs) were cultured on both patterned and control (non-patterned) polyacrylamide hydrogel matrices of $10 \mathrm{kPa}$ stiffness (Fig. 1). Cells cultured on fibronectin coated polyacrylamide can move readily and proliferate without impediment[51]. We chose to focus on MEFs because these cells are poised to proliferate and undergo morphogenetic transformations during normal development of the embryo. Polyacrylamide hydrogels were chosen because they can be fabricated to encompass numerous normal and pathological mechanical properties[52, 53]. Previous research relied on qualitative or fluorescent methods that do not quantify cellular mass accurately[54]. 
In order to quantify growth and transport characteristics, we seeded MEFs on patterned and control hydrogel substrates and imaged them with our GLIM system. The samples were imaged every thirty minutes for 100 hours. GLIM performs label-free measurements of optically thick samples and outputs quantitative phase gradient maps. We found that the patterned cells exhibit significant expansion and elevation as compared to the cells cultured on standard substrates (Fig. 2). By computing the cell height through a maximum average computation, we found that patterned cells reached a height of $20 \mu \mathrm{m}$ above their unpatterned counterparts. This behavior is captured quantitatively in Fig. 3. These data are consistent with previous work showing that designed substrate geometries induce differential and more robust growth tendencies[11].

\subsection{Patterned cells display inhomogeneous radial and rotational mass transport}

We applied a novel technique for investigating the dynamic properties of cellular transport. We calculated the standard deviation of the angular and radial velocity distribution for MEFs on patterned and unpatterned hydrogels. These parameters were obtained from the slope of the decay rate within the angular frequency range of $(0,10) \mathrm{rad} / \mathrm{rad}$, for rotational motion, and $(0,0.8) \mathrm{rad} / \mu \mathrm{m}$ for translational motion (Fig. 4). These intervals correspond to structures as large as the field of view, down to measurements as small as $0.314 \mathrm{rad}$ and $3.9 \mu \mathrm{m}$, respectively. Figure 5 shows an example of plots of polar DPS curves for both rotational and translational action. Curves of the rotational measurement show slopes in the range of $0.4-0.6 \mathrm{rad} / \mathrm{hr}$ and curves of the radial DPS measurement have slopes in the range of $10-12 \mu \mathrm{m} / \mathrm{hr}$, in the frequency range $\left(\mathrm{q}_{\theta}<10 \mathrm{rad} / \mathrm{rad}\right.$, $\left.\mathrm{q}_{R}<1 \mathrm{rad} / \mu \mathrm{m}\right)$. These findings are compatible with previous work indicating coordinated rotation of large population of cells when patterned in circular shapes[15] and with migration results of melanoma cells in our previous work[11].

\subsection{Testing the radial homogeneity and angular isotropy of cellular mass transport}


Next, we asked the question whether the angular velocity distribution changes with radial position.

Such variations would indicate that the cells produce shear stress between different concentric layers. Figure 6A shows that this is, in fact, not the case. The rotational velocity distribution width exhibits small fluctuations with the radius $\Delta v_{\theta}=\Delta v_{\theta_{0}} \pm \sigma_{\theta}=0.16 \pm 0.06 \mathrm{rad} / \mathrm{hr}$. The translational velocity distribution with respect to angle informs about the anisotropy of mass transport. Figure 6B indicates that there is no significant monotonic variation with angle: $\Delta v_{r}=\Delta v_{r_{0}} \pm \sigma_{r}=2 \pm 1.5 \mu \mathrm{m} / \mathrm{hr}$. These results hint at collective and harmonious cellular behavior that may imply emergent properties engendered through substrate patterning.

\section{Conclusion}

In this paper, we used label-free, high-throughput imaging to unravel unique growth and migration trends in 3D cellular systems, which are directed by initial conditions of the substrate mechanics and geometry. The combination of quantitative imaging with DPS in polar coordinates was used to quantify growth disparities caused by matrix confinements and to reveal specific cell movement patterns. MEFs cultured on engineered substrates exhibited greater out-of-plane growth. Cells situated along the edge of the culture disc showed angular velocity distributions similar to those closer to the center, which suggest that there is no significant shear stress along the radius. Rotational transport appears to be isotropic, consisted with the symmetry of the pattern.

We have demonstrated how combining quantitative phase imaging with specially designed substrates can determine variations in cell dynamics throughout 3D cultures, which may prove beneficial in studying spheroids, organoids, and microtumors. Future research will involve 
employing this methodology to study asymmetric substrate geometries and correlation between the dynamic parameters and cellular metastatic potential.

\section{Acknowledgements}

This work was supported by the National Institute of General Medical Sciences (NIGMS) grant

GM129709, the National Science Foundation (NSF) grant CBET-0939511 STC, DBI 14-50962

EAGER, IIP-1353368, as well as the (NSF)-funded Miniature Brain Machinery (MBM)

program. 


\section{References}

[1] C. A. Whittaker, K.-F. Bergeron, J. Whittle, B. P. Brandhorst, R. D. Burke, and R. O. Hynes, "The echinoderm adhesome," Developmental biology, vol. 300, no. 1, pp. 252-266, 2006.

[2] S. Özbek, P. G. Balasubramanian, R. Chiquet-Ehrismann, R. P. Tucker, and J. C. Adams, "The evolution of extracellular matrix," Molecular biology of the cell, vol. 21, no. 24, pp. 4300-4305, 2010.

[3] Y. Li, C. B. Tang, and K. A. Kilian, "Matrix Mechanics Influence Fibroblast-Myofibroblast Transition by Directing the Localization of Histone Deacetylase 4," Cellular and Molecular Bioengineering, vol. 10, no. 5, pp. 405-415, 2017.

[4] D. E. Ingber, "Cellular mechanotransduction: putting all the pieces together again," The FASEB journal, vol. 20, no. 7, pp. 811-827, 2006.

[5] N. Wang, J. P. Butler, and D. E. Ingber, "Mechanotransduction across the cell surface and through the cytoskeleton," Science, vol. 260, no. 5111, pp. 1124-1127, 1993.

[6] T. Chancellor, J. Lee, C. K. Thodeti, and T. Lele, "Actomyosin tension exerted on the nucleus through nesprin-1 connections influences endothelial cell adhesion, migration, and cyclic straininduced reorientation," Biophysical journal, vol. 99, no. 1, pp. 115-123, 2010.

[7] A. Degterev et al., "Chemical inhibitor of nonapoptotic cell death with therapeutic potential for ischemic brain injury," Nature chemical biology, vol. 1, no. 2, p. 112, 2005.

[8] J.-y. Wang, F. Xiao, Y.-p. Zhao, L. Chen, R. Zhang, and G. Guo, "Cell proliferation and thermally induced cell detachment of galactosylated thermo-responsive hydrogels," Carbohydrate Polymers, vol. 82, no. 3, pp. 578-584, 2010.

[9] D. B. Lovett, N. Shekhar, J. A. Nickerson, K. J. Roux, and T. P. Lele, "Modulation of nuclear shape by substrate rigidity," Cellular and molecular bioengineering, vol. 6, no. 2, pp. 230-238, 2013.

[10] A. S. Mao, J.-W. Shin, and D. J. Mooney, "Effects of substrate stiffness and cell-cell contact on mesenchymal stem cell differentiation," Biomaterials, vol. 98, pp. 184-191, 2016.

[11] Y. Li, M. J. Fanous, K. A. Kilian, and G. Popescu, "Quantitative phase imaging reveals matrix stiffness-dependent growth and migration of cancer cells," Scientific reports, vol. 9, no. 1, p. 248, 2019.

[12] C. M. Nelson et al., "Emergent patterns of growth controlled by multicellular form and mechanics," Proceedings of the National Academy of Sciences, vol. 102, no. 33, pp. 1159411599, 2005.

[13] E. W. Gomez, Q. K. Chen, N. Gjorevski, and C. M. Nelson, "Tissue geometry patterns epithelialmesenchymal transition via intercellular mechanotransduction," Journal of cellular biochemistry, vol. 110, no. 1, pp. 44-51, 2010.

[14] L. Q. Wan et al., "Geometric control of human stem cell morphology and differentiation," Integrative Biology, vol. 2, no. 7-8, pp. 346-353, 2010.

[15] K. Doxzen et al., "Guidance of collective cell migration by substrate geometry," Integrative biology, vol. 5, no. 8, pp. 1026-1035, 2013.

[16] K. Wolf et al., "Physical limits of cell migration: control by ECM space and nuclear deformation and tuning by proteolysis and traction force," J Cell Biol, vol. 201, no. 7, pp. 1069-1084, 2013.

[17] A. A. Abdeen, J. Lee, N. A. Bharadwaj, R. H. Ewoldt, and K. A. Kilian, "Temporal modulation of stem cell activity using magnetoactive hydrogels," Advanced healthcare materials, vol. 5, no. 19, pp. 2536-2544, 2016.

[18] N. D. Evans et al., "Substrate stiffness affects early differentiation events in embryonic stem cells," Eur cell mater, vol. 18, no. 1, p. e13, 2009.

[19] F. Pampaloni, E. G. Reynaud, and E. H. Stelzer, "The third dimension bridges the gap between cell culture and live tissue," Nature reviews Molecular cell biology, vol. 8, no. 10, p. 839, 2007. 
[20] K. M. Yamada and E. Cukierman, "Modeling tissue morphogenesis and cancer in 3D," Cell, vol. 130, no. 4, pp. 601-610, 2007.

[21] T. Chen, Y. You, H. Jiang, and Z. Z. Wang, "Epithelial-mesenchymal transition (EMT): a biological process in the development, stem cell differentiation, and tumorigenesis," Journal of cellular physiology, vol. 232, no. 12, pp. 3261-3272, 2017.

[22] A. Diaspro, Optical fluorescence microscopy: From the spectral to the nano dimension. Springer Science \& Business Media, 2010.

[23] R. Y. Tsien, "The green fluorescent protein," ed: Annual Reviews 4139 El Camino Way, PO Box 10139, Palo Alto, CA 94303-0139, USA, 1998.

[24] R. Hoebe, C. Van Oven, T. Gadella Jr, P. Dhonukshe, C. Van Noorden, and E. Manders, "Controlled light-exposure microscopy reduces photobleaching and phototoxicity in fluorescence live-cell imaging," Nature biotechnology, vol. 25, no. 2, p. 249, 2007.

[25] J. Lippincott-Schwartz, N. Altan-Bonnet, and G. H. Patterson, "Photobleaching and photoactivation: following protein dynamics in living cells," Nature Biotechnology, vol. 20, pp. 87-90, 2002.

[26] G. Popescu, Quantitative phase imaging of cells and tissues. McGraw Hill Professional, 2011.

[27] Y. Park, C. Depeursinge, and G. Popescu, "Quantitative phase imaging in biomedicine," Nature Photonics, vol. 12, no. 10, pp. 578-589, 2018/10/01 2018.

[28] B. Kemper et al., "Integral refractive index determination of living suspension cells by multifocus digital holographic phase contrast microscopy," J Biomed Opt, vol. 12, no. 5, p. 054009, Sep-Oct 2007.

[29] A. Calabuig, M. Mugnano, L. Miccio, S. Grilli, and P. Ferraro, "Investigating fibroblast cells under "safe" and "injurious" blue-light exposure by holographic microscopy," J Biophotonics, vol. 10, no. 6-7, pp. 919-927, Jun 2017.

[30] H. Ding, F. Nguyen, S. A. Boppart, and G. Popescu, "Optical properties of tissues quantified by Fourier-transform light scattering," Optics letters, vol. 34, no. 9, pp. 1372-1374, 2009.

[31] N. Lue, G. Popescu, T. Ikeda, R. R. Dasari, K. Badizadegan, and M. S. Feld, "Live cell refractometry using microfluidic devices," Optics letters, vol. 31, no. 18, pp. 2759-2761, 2006.

[32] G. Popescu, T. Ikeda, R. R. Dasari, and M. S. Feld, "Diffraction phase microscopy for quantifying cell structure and dynamics," Optics letters, vol. 31, no. 6, pp. 775-777, 2006.

[33] B. Bhaduri, K. Tangella, and G. Popescu, "Fourier phase microscopy with white light," Biomedical optics express, vol. 4, no. 8, pp. 1434-1441, 2013.

[34] B. Bhaduri, H. Pham, M. Mir, and G. Popescu, "Diffraction phase microscopy with white light," Optics letters, vol. 37, no. 6, pp. 1094-1096, 2012.

[35] Z. Wang et al., "Spatial light interference tomography (SLIT)," Optics express, vol. 19, no. 21, pp. 19907-19918, 2011.

[36] Z. Wang et al., "Spatial light interference microscopy (SLIM)," Optics express, vol. 19, no. 2, pp. 1016-1026, 2011.

[37] T. H. Nguyen, M. E. Kandel, M. Rubessa, M. B. Wheeler, and G. Popescu, "Gradient light interference microscopy for 3D imaging of unlabeled specimens," Nature communications, vol. 8, no. 1, p. 210, 2017.

[38] Y. Sung, W. Choi, C. Fang-Yen, K. Badizadegan, R. R. Dasari, and M. S. Feld, "Optical diffraction tomography for high resolution live cell imaging," Opt Express, vol. 17, no. 1, pp. 266-77, Jan 5 2009.

[39] W. Choi et al., "Tomographic phase microscopy," (in English), Nature Methods, vol. 4, no. 9, pp. 717-719, Sep 2007.

[40] F. Merola et al., "Tomographic flow cytometry by digital holography," (in English), Light-Science \& Applications, vol. 6, Apr 2017. 
[41] M. E. Kandel, D. Fernandes, A. M. Taylor, H. Shakir, C. Best-Popescu, and G. Popescu, "Threedimensional intracellular transport in neuron bodies and neurites investigated by label-free dispersion-relation phase spectroscopy," Cytometry Part A, vol. 91, no. 5, pp. 519-526, 2017.

[42] A. Vasdekis et al., "Eliciting the impacts of cellular noise on metabolic trade-offs by quantitative mass imaging," Nature communications, vol. 10, no. 1, p. 848, 2019.

[43] F. Zernike, "How I discovered phase contrast," Science, vol. 121, no. 3141, pp. 345-349, 1955.

[44] G. Nomarski, "Microinterféromètre différentiel à ondes polarisées," J. Phys. Radium, vol. 16, no. 3, pp. 9S-13S, 1955.

[45] R. Wang, Z. Wang, L. Millet, M. U. Gillette, A. J. Levine, and G. Popescu, "Dispersion-relation phase spectroscopy of intracellular transport," Opt. Express, vol. 19, no. 21, pp. 20571-20579, 2011.

[46] M. E. Kandel et al., "Label-free tissue scanner for colorectal cancer screening," Journal of biomedical optics, vol. 22, no. 6, p. 066016, 2017.

[47] M. E. Kandel, K. W. Teng, P. R. Selvin, and G. Popescu, "Label-free imaging of single microtubule dynamics using spatial light interference microscopy," ACS nano, vol. 11, no. 1, pp. 647-655, 2016.

[48] C. Hu and G. Popescu, "Quantitative Phase Imaging (QPI) in Neuroscience," IEEE Journal of Selected Topics in Quantum Electronics, vol. 25, no. 1, pp. 1-9, 2019.

[49] V. Venkatachalam, D. Schmidt, and M. Vander Heiden, "Development of a Method to Characterize the Effects of Radiation on Growth and Morphology of Organoids in 3D Culture," International Journal of Radiation Oncology• Biology• Physics, vol. 102, no. 3, pp. e154-e155, 2018.

[50] J. R. Tse and A. J. Engler, "Preparation of hydrogel substrates with tunable mechanical properties," Current protocols in cell biology, vol. 47, no. 1, pp. 10.16. 1-10.16. 16, 2010.

[51] T. Yeung et al., "Effects of substrate stiffness on cell morphology, cytoskeletal structure, and adhesion," Cell motility and the cytoskeleton, vol. 60, no. 1, pp. 24-34, 2005.

[52] J. Lee et al., "Melanoma topology reveals a stem-like phenotype that promotes angiogenesis," Science advances, vol. 3, no. 10, p. e1701350, 2017.

[53] J. Lee, A. A. Abdeen, X. Tang, T. A. Saif, and K. A. Kilian, "Matrix directed adipogenesis and neurogenesis of mesenchymal stem cells derived from adipose tissue and bone marrow," Acta biomaterialia, vol. 42, pp. 46-55, 2016.

[54] J. Lee, A. A. Abdeen, K. L. Wycislo, T. M. Fan, and K. A. Kilian, "Interfacial geometry dictates cancer cell tumorigenicity," Nature materials, vol. 15, no. 8, p. 856, 2016. 


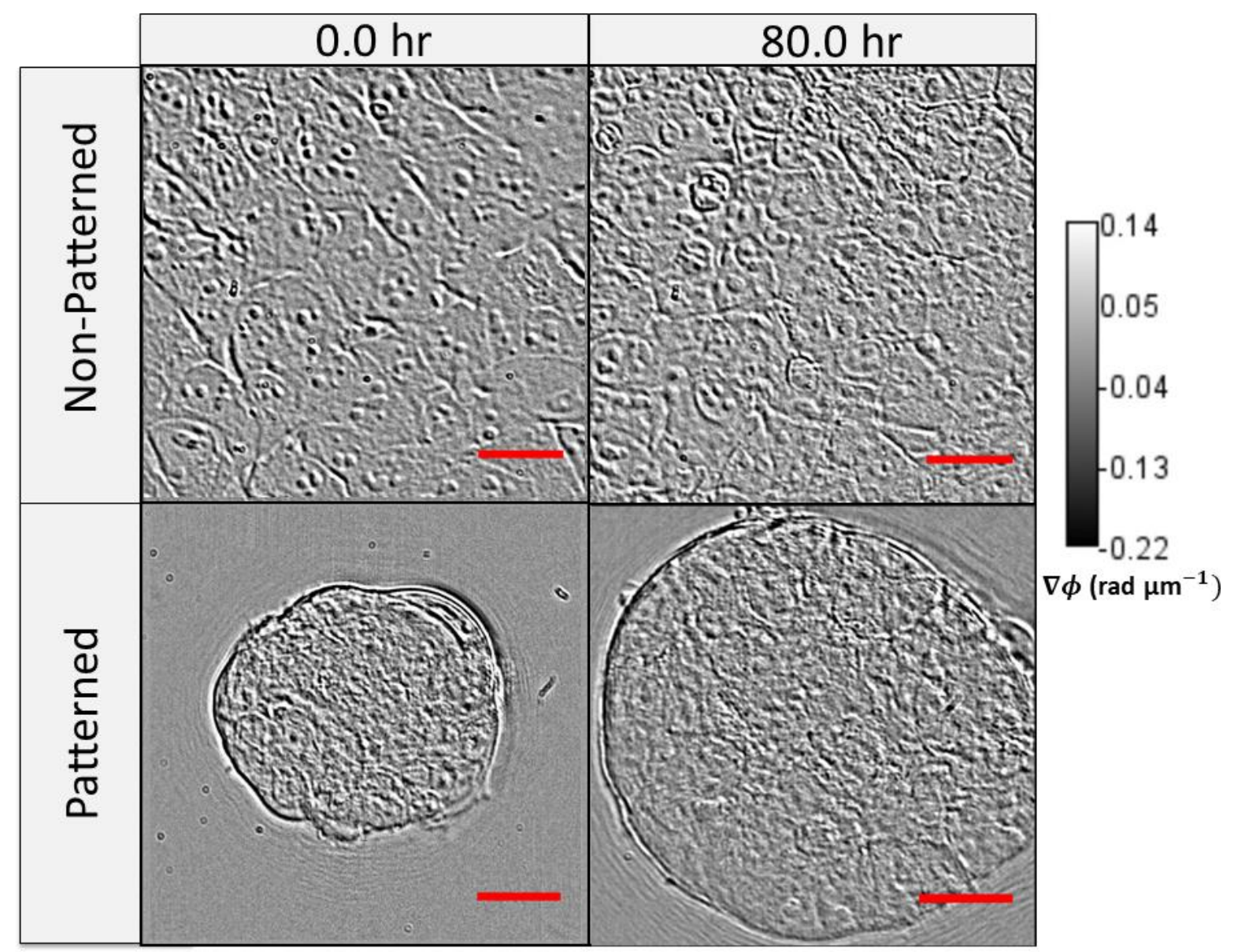

Figure 1. Gradient light interference microscopy (GLIM) images of mouse embryonic fibroblasts cultured on non-patterned and patterned substrates (polyacrylamide gels - $10 \mathrm{kPa}$ ), shown before and after 80 hours of growth. Scale bar: $30 \mu \mathrm{m}$. 


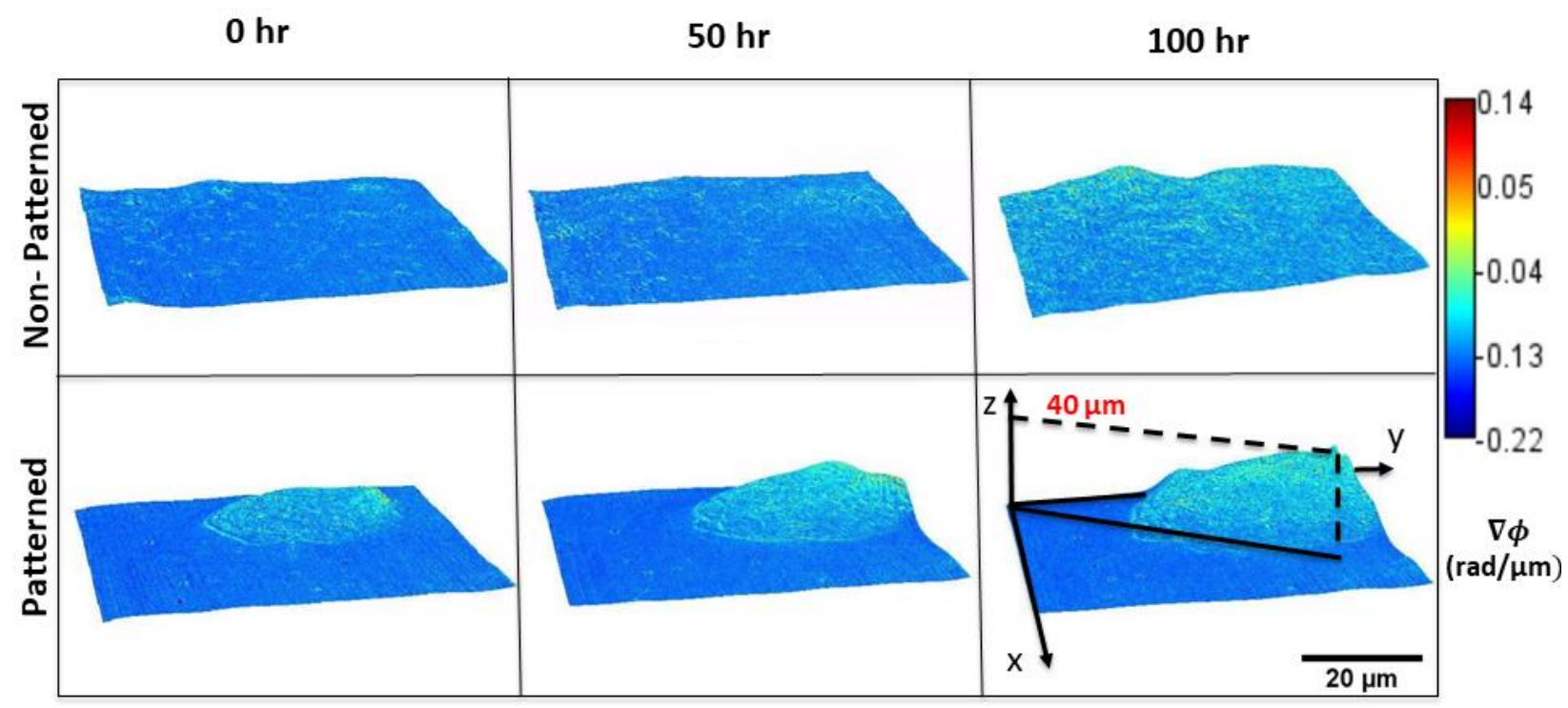

Figure 2. Gradient light interference microscopy (GLIM) images of mouse embryonic fibroblasts cultured on non-patterned and patterned substrates (polyacrylamide gels - $10 \mathrm{kPa}$ ), shown at 50 hour intervals. The cellular cluster on patterned substrates exhibits lateral growth while rising and rotating about the vertical axis. 

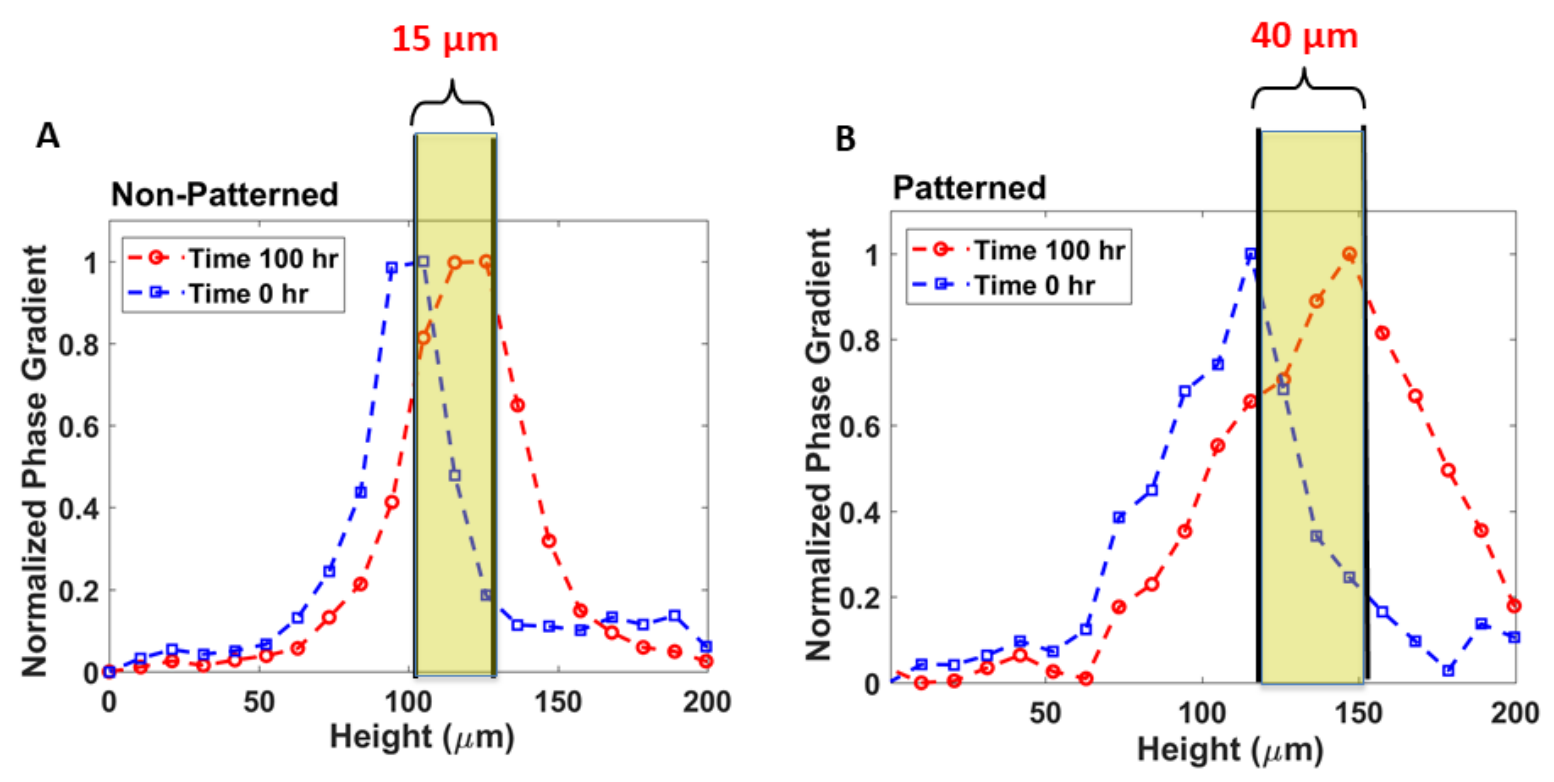

Figure 3. Total substrate height determined from peak maximum values of normalized phase gradients for non-patterned substrates indicate a $15 \mu \mathrm{m}$ height increase over 100 hours (A). Total substrate height determined from the peak maximum values of normalized phase gradients for patterned substrates indicate a $40 \mu \mathrm{m}$ height increase over 100 hours (B). 

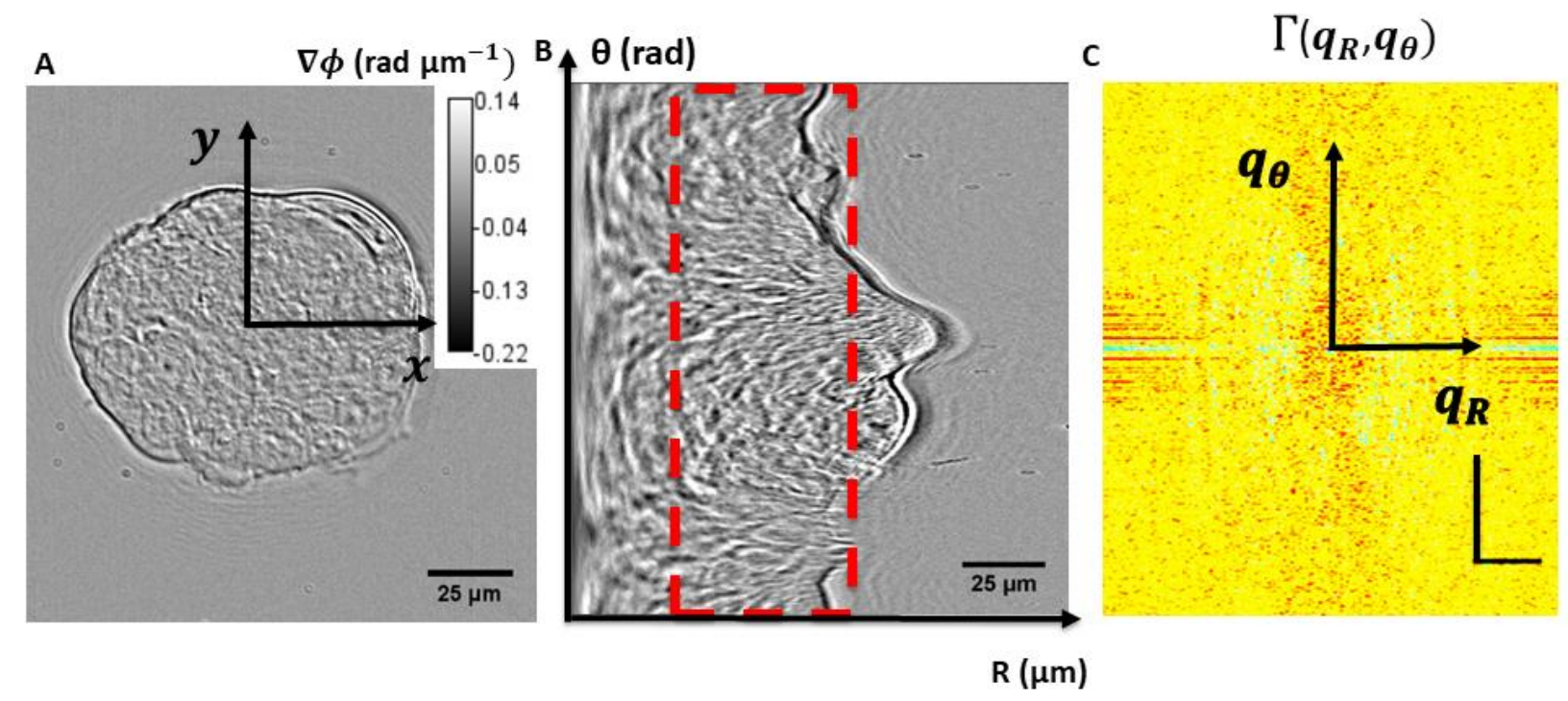

Figure 4. GLIM image of mouse embryonic fibroblasts cultured on a patterned substrate (A), and the corresponding polar transformation of the image, with an angular vertical dimension and a horizontal radial dimension, and showing the region or "ring" (red dashed rectangle) that was used in the computation (B). Decay rate vs spatial mode (angular and radial) associated with polar GLIM images (horizontal scale bar: $20 \mathrm{rad} / \mu \mathrm{m}$; vertical scale bar: $20 \mathrm{rad} / \mathrm{rad}$ ) (C). 

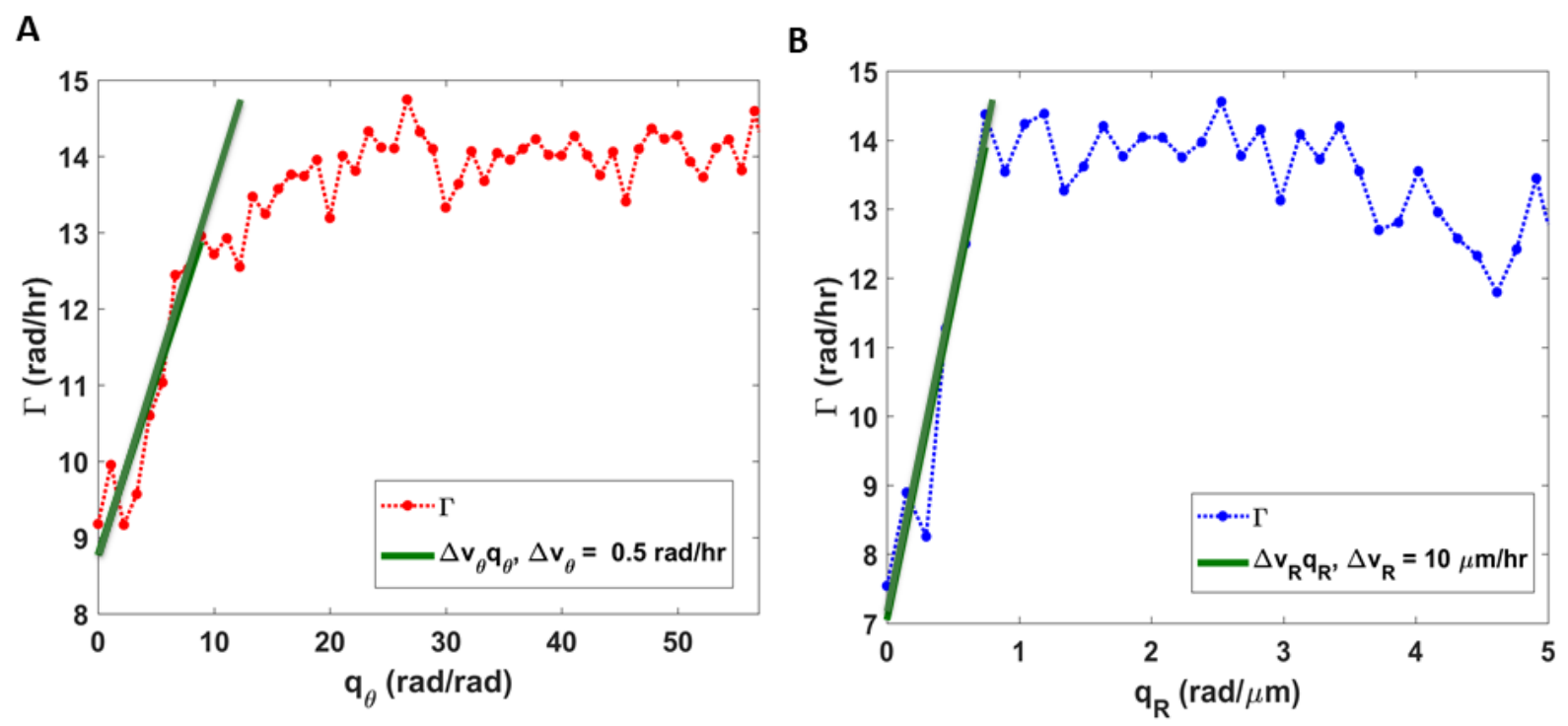

Figure 5. Angular decay rate with a slope of the linear fit down to 0.34 radians, indicating the angular velocity distribution width about a certain diameter $(56 \mu \mathrm{m})(\mathrm{A})$. Radial decay rate with a slope of the linear fit down to $3 \mu \mathrm{m}$, indicating the radial velocity distribution width around the centroid of the patterned substrate $(\mathrm{B})$. 
A

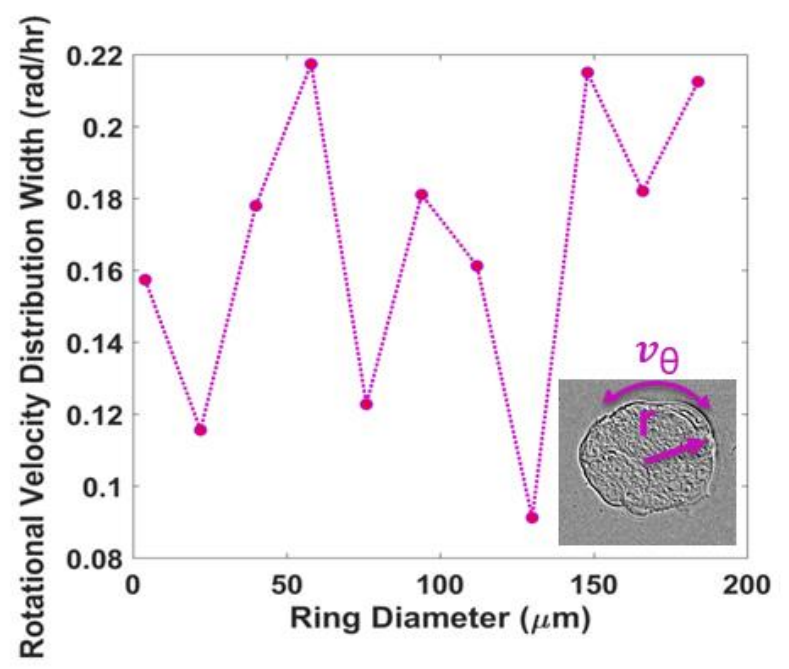

B

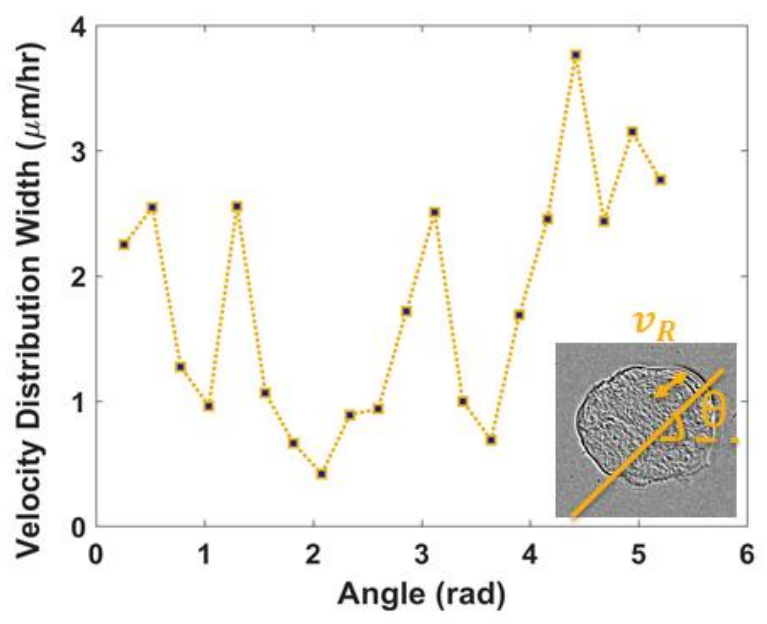

Figure 6. Rotational velocity distribution width of rings of the patterned substrate at varying inner diameters, with a ring thickness of $28 \mu \mathrm{m}$ (A). Velocity (radial) distribution width at different angles (B). 